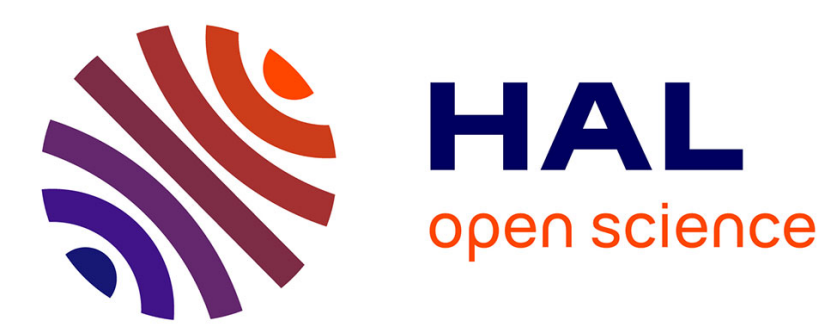

\title{
Quel partage des responsabilités dans la gestion de l'eau souterraine en Inde du Sud? (cas du Tamil Nadu et de Pondichéry)
}

Olivia Aubriot

\section{- To cite this version:}

Olivia Aubriot. Quel partage des responsabilités dans la gestion de l'eau souterraine en Inde du Sud? (cas du Tamil Nadu et de Pondichéry). Science et changements planétaires / Sécheresse, 2010, 21 (1), pp.28-33. 10.1684/sec.2010.0225 . halshs-01694868

\section{HAL Id: halshs-01694868 \\ https://shs.hal.science/halshs-01694868}

Submitted on 13 Feb 2018

HAL is a multi-disciplinary open access archive for the deposit and dissemination of scientific research documents, whether they are published or not. The documents may come from teaching and research institutions in France or abroad, or from public or private research centers.
L'archive ouverte pluridisciplinaire HAL, est destinée au dépôt et à la diffusion de documents scientifiques de niveau recherche, publiés ou non, émanant des établissements d'enseignement et de recherche français ou étrangers, des laboratoires publics ou privés. 


\section{Quel partage des responsabilités} dans la gestion de l'eau souterraine
en Inde du Sud?

\section{(cas du Tamil Nadu et de Pondichéry)}

\section{Olivia Aubriot}

\section{CNRS}

UPR 299, Centre d'études himalayennes

7, rue Guy Môquet

94801 Villejuif cedex

France

<oaubriot@vif.cnrs.fr>

\section{Résumé}

Cet article traite de l'absence de gestion coordonnée de l'irrigation par eau souterraine en Inde du Sud, I'utilisation de la ressource étant le fait de millions d'individus indépendants y ayant accès librement. II soulève la question du rôle de l'État et du partage des responsabilités entre un État ayant favorisé le développement de ce type d'irrigation sans en restreindre l'usage, des agriculteurs pompant tant et plus, toujours plus profondément, et la société civile partie prenante dans les choix d'évolution de la société et de protection de l'environnement. Des exemples tirés de travaux scientifiques et d'observations réalisées dans la région de Pondichéry illustrent les réactions des différents acteurs envers la crise de l'eau que traverse le pays, et notamment l'État du Tamil Nadu. Les solutions résideraient dans une clarification des droits d'appropriation de l'eau souterraine et dans une régulation de l'exploitation de cette ressource sous forme de gestion communautaire (avec certaines limites en termes de justice sociale et d'intégration des échelles et des secteurs) ou de gestion concertée.

Mots clés : eau souterraine, gestion de l'eau, Inde.

\section{Abstract}

Sharing responsibility for groundwater management in South India: Cases from Tamil Nadu and Pondicherry

This paper addresses the lack of coordinated management of groundwater irrigation in South India brought about by the fact that the resource is used by millions of independent individuals who have free access to it. The main issues are the involvement of the State and the shared responsibility between: i) a state who has always given incentives to this type of irrigation without imposing any restrictions; ii) farmers pumping more and more water; and iii) civil society involvement in the environmental and social debate. Examples from the literature and observations of Pondicherry area illustrate the way various stakeholders react to the water crisis affecting the country and especially the Southern state of Tamil Nadu. Solutions could be implemented through a clarification of property rights and regulation of water use under a community management or a dialogue and cooperation process between state agencies, NGOs and communities.

Key words: groundwater, India, water management. 
"Gérer l'anarchie avec laquelle l'irrigation par eau souterraine s'est développée est l'un des défis de l'Asie du Sud » : ainsi commence l'article de Shah [1], I'un des économistes libéraux indiens les plus renommés pour traiter de l'irrigation par eau souterraine. Les mots sont forts, et sous-tendent que la gestion - ou plutôt la non-gestion - de cette ressource est un problème qui n'est pas simple à résoudre.

Le développement de ce mode d'irrigation, qui alimente aujourd'hui la majorité des terres irriguées (près de $60 \%$ [2], voire les trois quarts [3] selon les sources), elles-mêmes représentant $30 \%$ de la superficie cultivée, est en effet le fait de millions d'individus indépendants. L'eau souterraine est ainsi devenue primordiale dans l'agriculture indienne. Mais l'accès libre dont bénéficient les propriétaires des 19 millions de puits a provoqué en maints endroits une surexploitation des nappes dont nous allons présenter les principaux impacts socio-économiques et environnementaux. Qui est responsable de cette situation conduisant à une véritable crise de l'eau, à une pénurie "créée par l'homme » disent certains et qui rappelle, du fait du libre accès, la «tragédie des communaux " décrite par Hardin en 1968 et prévoyant un épuisement de la ressource ainsi exploitée $[4,5]$ ? Est-ce l'État, promoteur du développement de l'irrigation souterraine au titre des réformes de la révolution verte, ou les agriculteurs, consommant $80 \%$ de l'eau captée [6] et traités par certains d'usagers sans scrupules? A qui incombe la responsabilité, de gérer durablement cette ressource? À l'État, garant de la gestion des ressources collectives $^{1}$, aux agriculteurs, premiers touchés par une dégradation de l'accès aux nappes, ou à la société civile ${ }^{2}$ (désignant ici les Organisations non gouvernementales (ONG), les chercheurs et les militants des mouvements environnementaux et sociaux), parties prenantes dans les choix d'évolution de la société ?

\footnotetext{
${ }^{1}$ La terminologie relative aux ressources communes prête à confusion car les adjectifs public, collectif, commun, privé sont utilisés pour qualifier des éléments différents tels que l'appropriation, le régime de consommation et le mode de gouvernance du bien [7]. Une acception simplifiée du terme collectif - qui relève principalement de l'État - est employée ici, mais la question des régimes de propriété est abordée plus loin.

2 L'utilisation du terme "société civile» peut être débattue dans la mesure où en Inde, de nombreuses organisations sont fortement liées au domaine politique. Ainsi, les hommes politiques, inclus dans notre catégorie "État», peuvent faire partie de la société civile. On pourrait également leur créer une catégorie à part entière.
}

Après avoir présenté le contexte du développement de l'irrigation en Inde, nous brosserons un tableau général des réponses apportées jusqu'à présent par les principaux acteurs.

\section{Un développement non durable de l'utilisation de l'eau}

\section{Développement spectaculaire de l'irrigation par eau souterraine}

L'irrigation par l'eau souterraine est ancienne en Inde, mais elle a connu son essor avec la motorisation des puits promue depuis le milieu des années 1960 par les politiques de la révolution verte. Ainsi, de 1950 à 2005, le nombre de pompes motorisées est passé de moins de 100000 à près de 19 millions (la majorité des puits en étant dorénavant équipés) et la superficie ainsi irriguée de 6 à 46 millions d'hectares [8] $]^{3}$.

Deux phases principales s'observent dans le développement de cette irrigation. La première est celle du lancement des politiques destinées à la favoriser : électrification de la campagne, électricité subventionnée, prêts bonifiés pour la construction de puits et l'achat de pompes. Les nombreux avantages de cette irrigation expliquent l'engovement des agriculteurs à investir dans cette nouvelle technique. L'irriguant se libère des contraintes climatiques, intensifie son calendrier cultural en cultivant en saison sèche, transforme des terres pluviales en terres irriguées et ne connaît aucune restriction d'usage hors coupures d'électricité ou pannes de moteur.

À la fin des années 1970, les pompes submersibles sont introduites en milieu agricole. Ainsi, des profondeurs plus importantes que la vingtaine de mètres autorisés par les pompes aspirantes sont atteintes et l'eau peut être pompée à de grandes profondeurs. Une nouvelle ère s'instaure, celle des forages, dorénavant omniprésents en zones alluviale et sédimentaire.

Pour un État qui, à partir de 1976, se dit socialiste, soutenir une telle irrigation présente plusieurs avantages: augmentation de la production et des superficies irriguées (figure 1), accession des petits propriétaires fonciers à cette ressource à travers des programmes d'aide spécifique, lutte contre la pauvreté en abaissant le seuil de viabilité des petites exploitations.

\footnotetext{
${ }^{3}$ National Sample Survey Organisation (NSSO). Ministry of Statistics, 2007. www.indiastat.com/ agriculture/2/irrigation/145/stats.aspx
}

Un point essentiel est à souligner : le droit d'extraction de l'eau souterraine est lié à la propriété foncière, sans limitation de quantité ou de profondeur. Cette eau souterraine est alors considérée par les agriculteurs comme un bien privé - ou du moins comme une ressource sur laquelle le propriétaire foncier a un droit d'usage non limité - tout en reconnaissant que les nappes sont des entités collectives. On voit ici l'ambiguité des droits d'appropriation de cette eau: il s'agit d'une ressource commune qui, juridiquement, $n^{\prime}$ appartient à personne (res nullius), que l'État central a la charge de protéger et pour laquelle le régime de propriété ne correspond à aucun des quatre types définis par Bromley [9], et relatifs au sujet détenant ce droit (propriété publique, propriété privée, propriété commune et libre accès où aucun droit ni devoir n'est établi) [cité par 5]. La situation indienne apparaît plus complexe. Aucune régulation de l'usage de l'eau n'existant, les utilisateurs ont tendance à confondre leur droit de propriété foncière - qui leur confère un droit d'aliénation du sol, auquel le droit d'extraire l'eau est lié - et leur droit d'usage de la ressource, puisqu'ils peuvent vendre l'eau qu'ils extraient. Des marchés informels de l'eau se sont en effet développés, qui ont favorisé l'essor de l'irrigation par eau souterraine. Ils permettent à ceux qui n'ont pas les moyens d'investir dans un puits ou forage $d^{\prime}$ 'avoir accès à cette ressource, et aux vendeurs d'eau - pour qui le pompage a un faible coût du fait de l'électricité subventionnée - de rentabiliser leurs infrastructures.

La loi établissant la relation entre droit d'extraction de l'eau et propriété foncière remonte à 1882 (Easement Act [10]), époque où seuls des puits d'une dizaine de mètres de profondeur existaient, sans moteur. Or, le progrès des moyens d'exhaure a modifié les conditions d'exploitation des nappes, mais n'a pas été accompagné d'une évolution des lois.

\section{Surexploitation des nappes}

L'exploitation croissante des différentes nappes souterraines a progressivement conduit à une baisse de leur niveau et à l'épuisement rapide de la nappe superficielle après la saison des pluies. Ainsi, à l'échelle de l'Inde, $15 \%$ des unités administratives sont considérées comme "surexploitées », c'est-à-dire que la quantité d'eau pompée est supérieure au réapprovisionnement naturel de ces nappes (par infiltration des eaux de pluies, d'irrigation, de lacs, etc.). Les zones surexploitées sont toutefois concentrées dans cinq États, et en Inde du Sud au seul Tamil Nadu, dont $37 \%$ des unités administratives de gestion des projets ou «blocs » sont ainsi catalogués [8]. Le développement de l'irrigation 


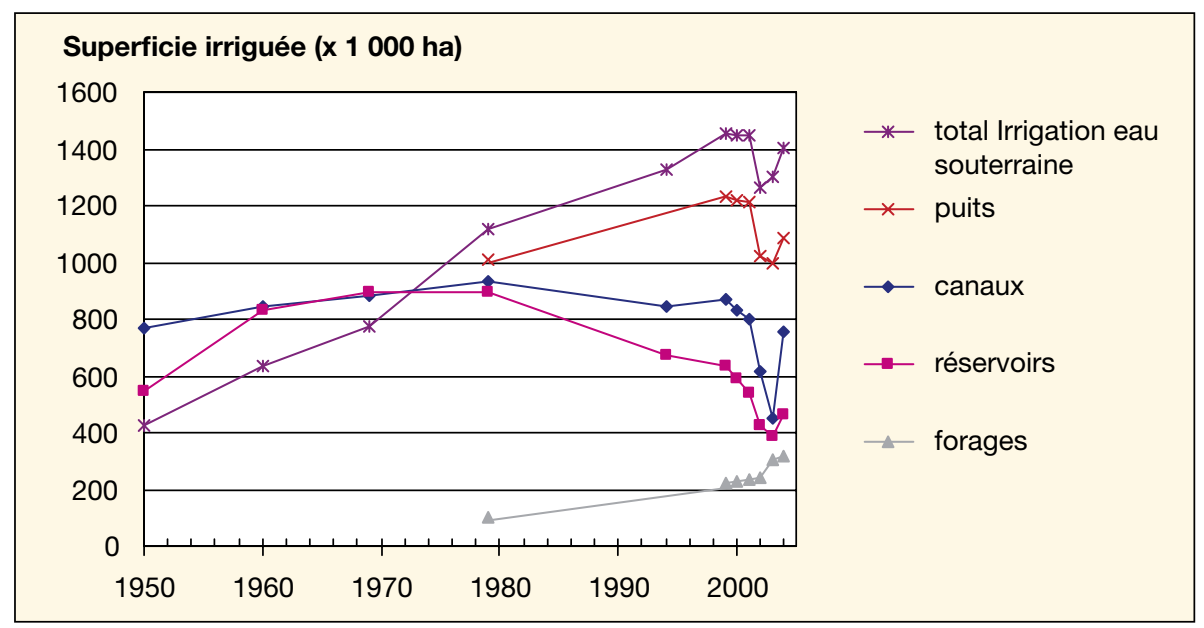

Figure 1. Évolution des superficies irriguée (nettes) au Tamil Nadu, par type d'irrigation.

par eau souterraine y a provoqué le déclin, voire l'abandon ${ }^{4}$, des systèmes d'irrigation ancestraux, collectifs, par étangs-réservoirs temporaires. Ainsi, dans la zone alluviale de la région de Pondichéry (environ $600 \mathrm{~km}^{2}$ ), la majorité des villages ont abandonné leur étang et dépendent dorénavant exclusivement de l'eau souterraine pour tous leurs usages: irrigation, industrie et besoins domestiques.

D'un point de vue environnemental, la baisse du niveau des nappes est en soit problématique. En outre, elle s'accompagne souvent d'une dégradation de la qualité de l'eau en raison de processus de déminéralisation et, en zone côtière, $d^{\prime}$ 'intrusion d'eau saline, aux répercussions sanitaires inquiétantes car affectant l'eau à usage domestique. Quant aux conséquences socio-économiques, elles sont liées au surcreusement des puits et forages: augmentation du coût $d$ 'installation, inégalité croissante de l'accès à l'eau puisque ceux qui $n^{\prime}$ ont pas les moyens de surcreuser leur ouvrage se retrouvent sans eau. L'assèchement des puits peu profonds touche principalement les petits agriculteurs qui sont les principaux détenteurs de ces infrastructures [13, 14]. Une véritable crise de l'eau souterraine est amorcée.

\section{Les réponses à cette crise de l'eau}

\section{Un État fournisseur et non protecteur de la ressource}

L'État central de l'Union indienne, République fédérale, oriente la politique générale et chaque État formule ensuite ses

\footnotetext{
${ }^{4}$ Les raisons de ce déclin sont multiples. Cf Janakarajan [11] et Mosse [12] pour une synthèse.
}

propres lois et politiques de l'eau selon $d^{\prime}$ interventions se distinguent dans les politiques publiques indiennes relatives à leau souterraine : la régulation directe à travers des lois, la régulation indirecte à travers des taxes et subventions, et l'investissement public dans des infrastructures de production [15].

\section{- Régulation directe}

En 1970, 1992, 1996, et 2005, l'État central présenta des directives, mais peu d'États ont édicté leur propre loi. Le Tamil Nadu l'a fait en 2003, mais n'a toujours pas établi de décret d'application. Pourtant, réguler l'extraction de l'eau semble indispensable, "la situation de l'eau souterraine dans cet État étant l'une des pires du pays » [8]. D'après les textes, les nouveaux puits devront être enregistrés et dans les zones répertoriées comme critiques un permis d'utilisation de l'eau sera requis, spécifiant la localisation du puits, le nombre d'heures de pompage, la période $d^{\prime} u_{i}$ ilisation ${ }^{5}$. L'établissement de nouveaux forages ou le surcreusement dans les zones classées "surexploitées » ne seront pas autorisés. Cette régulation nécessite, pour être effective, de disposer d'une agence de contrôle. Or une telle surveillance est difficile à réaliser auprès de millions de propriétaires, et cette fonction ne relève pas du Bureau central des eaux souterraines (Central Ground Water Authority) créé en 1997, dont les seules missions sont de notifier les zones à protéger et de conduire des programmes $d^{\prime}$ information sur la récupération de l'eau de pluie [3].

L'approche par une régulation purement réglementaire est très critiquée, car ayant jusqu'à présent eu peu d'impacts réels.

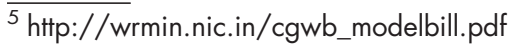
les directives promulguées. Trois types
Ainsi, au Tamil Nadu, une distance minimum entre les puits est requise pour ceux qui demandent un prêt gouvernemental. Or la majorité des agriculteurs utilisant du crédit non institutionnel, la règle n'est pas suivie [16]. L'État d'Andhra Pradesh a passé en 2002 une loi de régulation de l'exploitation de l'eau souterraine, mais dès que la saison sèche arrive, la loi n'est plus appliquée [14]. "Même là où une législation sur l'eau souterraine existe, elle reste largement un exercice écrit » [3].

\section{- Régulation indirecte}

Un autre moyen de réguler le développement de cette irrigation est d'agir sur les facteurs de production. Le prix de l'électricité est à ce titre le plus sujet à controverse, cette énergie étant fortement subventionnée, voire gratuite, comme au Tamil Nadu depuis 1991. Certains prônent la solution de faire payer davantage tout en assurant la qualité d'approvisionnement, ce qui favorisera une utilisation plus efficiente de l'eau [17], alors que d'autres concluent qu'une politique fondée uniquement sur le prix de l'énergie n'aura pas les effets escomptés: rationner les heures de délivrance de l'électricité, fixer des tarifs différenciés, améliorer l'efficacité des pompes et sensibiliser conjointement aux effets de la surexploitation des nappes, aurait davantage d'impacts [18].

Le deuxième type d'influence indirecte concerne les aides à l'équipement hydraulique. Ces mesures sont plus ou moins développées et efficaces. Le district de Pondichéry par exemple est dorénavant équipé de nombreuses installations de tuyaux enterrés, mais très peu en matériel de micro-irrigation. Certes, subventionner ces infrastructures utilisées uniquement avec de l'eau pompée signifie reconnaître l'importance et la dépendance vis-à-vis de cette ressource. En revanche, dans le cas de la canne à sucre, irriguée uniquement avec cette eau, de telles installations s'avèrent indispensables pour réduire la consommation.

Le troisième moyen consiste à inciter à modifier les cultures pratiquées, en subventionnant celles qui consomment moins d'eau, comme le fait le gouvernement de Pondichéry depuis 2006 pour la culture de légumineuses en saison sèche. Les cultures les plus rentables ou culturellement importantes en Inde du Sud sont de fait la canne à sucre et le riz, très consommatrices d'eau.

Enfin, un quatrième moyen est de favoriser des pratiques culturales économisant l'eau, tel le SRI (system of rice intensification) qui permet jusqu'à $30 \%$ de réduction d'eau [19]. Le pays, et l'Asie en général, va donc devoir trouver une façon de produire plus de riz en consommant moins d'eau [20]. 
- Investissement dans des infrastructures collectives et implication des villageois

Pour pallier le manque d'eau, I'Inde a fait le choix de privilégier la récupération des eaux de pluie et la recharge des nappes. Au Tamil Nadu, une loi oblige à installer des infrastructures de récupération de l'eau de pluie, mais les bâtiments publics ne sont pas eux-mêmes systématiquement aménagés. Les projets de bassin-versant (à travers divers ministères et $\mathrm{ONG}$ ) ont également une grande part de leur activité orientée vers la construction de retenues collinaires, le nettoyage des marres ou autres ouvrages de stockage de l'eau de pluie, et ce en impliquant des villageois organisés en associations d'utilisateurs de l'eau. Mais ces programmes de gestion participative ne concernent que l'eau de surface, à l'exception de l'un d'eux qui s'intéresse à l'eau souterraine à usage domestique et met l'accent sur la concertation, l'information et la prise de conscience, processus assez exceptionnel, nouveau et innovant pour être notéb.

L'État continue à s'installer dans un rôle de fournisseur d'eau à travers des techniques modernes (barrages, pompes, forages) et des programmes de recharge des nappes, et ne se positionne pas comme protecteur de la ressource [22]. De manière générale, très peu de mesures concernent l'économie d'extraction d'eau ou la gestion collective de la ressource souterraine. Et les hommes politiques $\mathrm{n}^{\prime}$ osent pas prendre de mesures restrictives, trop impopulaires.

\section{Concurrence effrénée entre agriculteurs pour l'approfondissement des puits et forages}

À la baisse du niveau des nappes, les agriculteurs répondent en surcreusant leur puits ou en forant davantage ef plus profondément ${ }^{7}[23]$, notamment en période de sécheresse, comme le prouve l'augmentation de la superficie ainsi

\footnotetext{
${ }^{6}$ Le bureau chargé de l'eau domestique en milieu rural (TWAD, Tamil Nadu Water Supply and Drainage) a lancé en 2003 un projet pilote de "démocratisation de la gestion de l'eau " dans 200 villages de l'État, appliquant ainsi les nouvelles perspectives de la gouvernance: des forums sont organisés avec les communautés où la responsabilité des villageois dans la crise de l'eau et dans les possibilités de la résoudre sont discutés [21]. Ce projet est mené dans l'optique de répondre à une nouvelle gouvernance locale qui permettra à l'État de se désengager financièrement: l'eau potable sera bientôt gérée par les collectivités locales, avec les risques de manques de moyens financiers et de déresponsabilisation de l'État que cela comporte.
}

irriguée au Tamil Nadu de 2001 à 2003 (figure 1). Cette quête de l'eau est accentuée par le statut social induit par la possession d'un puits/forage [23], et notre analyse d'une cinquantaine de forages dans un village près de Pondichéry montre clairement que la profondeur du forage reflète les différences socio-économiques. Si la terre représentait autrefois l'élément de distinction par excellence, aujourd'hui la détention d'un forage [13] ainsi que sa profondeur joue davantage ce rôle.

En zone rocheuse où seule la nappe superficielle est accessible et les forages peu présents car trop onéreux et risqués, les enquêtes de terrain ${ }^{8}$ révèlent que tous les agriculteurs ont surcreusé d'une douzaine de mètres leurs larges puits et les ont équipés de galeries drainantes.

La première réaction des propriétaires de forages à la baisse de production d'une nappe est de réduire la vente d'eau. En effet, dans la région étudiée, les propriétaires de puits et forages ne changent pas leur propre utilisation de l'eau, mais ils réduisent le nombre d'acheteurs tout en augmentant le prix de l'eau. Ceux qui ne peuvent acheter l'eau quittent l'agriculture ou se remettent à cultiver en pluvial, agriculture peu rentable et risquée $[13,24]$. Les marchés informels de l'eau tant prônés par les économistes libéraux $[15,17]$ tendent à diminuer et trouvent alors leurs limites dès que la ressource est moins disponible.

La course effrénée pour l'approfondissement des puits et forages est décrite par de nombreux auteurs $[10,13,14,24]$. Ceux-ci citent les cas étonnants de larges puits à l'intérieur desquels des murs ont été érigés pour délimiter matériellement la part de chaque héritier. Et bien sûr, l'approfondissement de l'une des parties engendre l'assèchement des autres ! De même, l'installation d'un forage assèche progressivement les puits qui l'entourent. Or ces phénomènes sont acceptés par les agriculteurs car l'eau est ici considérée comme un bien privé. Il est intéressant de comparer cette perception au cas de l'usine Coca Cola dans l'État du Kérala, qui s'est vu imposer la fermeture pour cause $d$ 'assèchement

des puits environnants : I'appropriation du bien, alors présenté comme public par les militants environnementalistes et sociaux,

\footnotetext{
$\overline{7}$ Ainsi, dans un village de la plaine alluviale de la région de Pondichéry, le niveau moyen de pompage de l'eau était de 18 mètres dans les années 1970, il est aujourd'hui de 70 mètres. Les agriculteurs les plus aisés pompent également dans cette nappe, mais leur forage est profond de 150 mètres, ainsi mis à l'abri d'une baisse accrue éventuelle du niveau de l'eau.

${ }^{8}$ Dans les districts de Pondichéry (Territoire de Pondichéry) et Villupuram (État du Tamil Nadu).
}

par une multinationale aux dépens des populations locales a été l'objet d'un conflit virulent $[8,25]$.

Les agriculteurs indiens sont lancés dans une course effrénée au pompage. II ressort indéniablement de nos entretiens ${ }^{9}$ et des travaux consacrés à ce sujet que les agriculteurs n'ont pas le choix: il faut investir dans un puits/forage, "do or die " [14]. Les irriguants sont conscients de la baisse du niveau des nappes, de l'augmentation du coût de l'irrigation par forage, mais tant que l'eau sort du tuyau, ils continuent à pomper. L'idée d'économiser l'eau aujourd'hui pour en avoir demain n'est pas dans leurs préoccupations. Cela est certes lié au fait que la ressource est invisible mais ces pratiques soulèvent également la question de la perception de la rareté de l'eau [26,27], celle de la méconnaissance par les agriculteurs du cycle de l'eau et de l'absence de quantification de l'eau pompée, points qu'il serait trop long de développer ici. Par ailleurs, accuser l'insuffisance des pluies [23] ou encore les usines pompant de larges quantités d'eau [28] est aussi une façon pour les agriculteurs de se déresponsabiliser de la baisse des nappes.

\section{Une société civile faiblement investie} dans ce champ d'action

Nombreuses sont les ONG impliquées dans des projets de recharge des nappes et de réhabilitation des étangs-réservoirs traditionnels, mais n'ayant pas de légitimité à agir sur une ressource dont l'exploitation est liée à la propriété foncière individuelle, très peu s'occupent de minimiser l'utilisation de l'eau souterraine. Quelques cas de puits collectifs ou de mobilisation autour de la préservation des ressources existent notamment l'exemple du Pani Panchayat créé en 1972 dans l'État du Maharastra [18] ou des cas dans les régions désertiques du Gujarat et du Rajasthan, " efforts sporadiques et de faible échelle d'impact » [29].

\section{La solution réside-t-elle dans une gestion concertée?}

Les régulations directes se révélant inefficaces, les régulations indirectes encore insuffisantes, et les investissements dans les infrastructures collectives favorisant I'utilisation d'eau souterraine, quelle nouvelle forme de gestion permettrait de remédier à la crise de l'eau souterraine que

\footnotetext{
${ }^{9}$ Plus d'une centaine d'entretiens ont été réalisés entre septembre 2005 et septembre 2007.
} 
connaît l'Inde? Trois types de solutions non exclusifs les uns des autres - se dégagent de la littérature.

\section{Changement de régime de propriété de l'eau souterraine}

Ce premier type de solutions est demandé par de nombreuses associations. L'eau doit devenir "une ressource commune, gérée par des communautés ", pour pallier la privatisation de l'eau qui émerge à travers le pays et qui permet à tous les propriétaires fonciers, État compris, de surexploiter la ressource [30]. Notons ici la confusion entre la nature de la ressource (l'eau est de toute manière un bien commun) et le régime de propriété (sousentendu un régime de propriété commune). Le rapport à mi-parcours de la commission du $10^{\mathrm{e}}$ plan quinquennal propose que la question de la propriété de 'eau soit l'objet d'un débat public, deux solutions étant suggérées : dans l'une, la nappe superficielle reste attachée au sol et les nappes profondes seraient des ressources communautaires; dans l'autre, le propriétaire foncier utilise la quantité d'eau qui lui est nécessaire, le restant devant être une ressource communautaire [3]. Là encore, l'ambiguïté des droits d'appropriation de l'eau souterraine en Inde rend confuse la distinction entre, d'une part, régime de propriété de l'eau lassocié au régime de propriété foncière ou passant sous un régime de propriété commune) et, d'autre part, droit d'usage (sous-entendu limité aux nécessités du propriétaire foncier). À l'inverse, malheureusement, le rapport d'experts de 2007 suggère de ne pas modifier les droits d'appropriation de l'eau, précisant que « les droits d'extraction peuvent et doivent être contrôlés par l'État si l'utilisation de l'eau est considérée excessive » [8], dissociant donc clairement régime de propriété et droit de gestion de l'eau, l'État devant intervenir. Cette suggestion va dans le sens des récentes réformes sur l'eau (National Water Policy, 2002 ; Groundwater Modell Bill, 2005), qui redéfinissent le rôle du gouvernement dont la responsabilité est amoindrie mais la fonction de contrôle sur la gestion de la ressource renforcée [31]. Elle laisse en outre une zone trouble autour de la notion « d'excessif ».

\section{Création d'associations \\ pour limiter l'utilisation de l'eau et gérer la demande}

C'est le deuxième type de solutions. "Des associations d'usagers de l'eau souterraine existent et fonctionnent relativement bien aux États-Unis, Mexique et France » [32]. Plusieurs éléments expliquent les réti- cences qu'a rencontrées cette solution en Inde jusqu'ici, le dernier rapport d'experts de la commission montrant toutefois un changement de position. L'autorégulation et la pression sociale comme moyen de contrôle ne peuvent fonctionner pour l'eau souterraine car la communauté n'est pas vulnérable à la disparition de cette ressource de façon homogène [1]. Le fonctionnement encore très hiérarchique et inégalitaire de cette société de castes combiné à la prégnance du domaine politique rend effective l'inégalité d'accès aux positions décisionnelles et limite les possibilités de pression sociale par toute une catégorie de la population. En outre, le nombre de personnes impliquées serait rapidement très important et difficile à organiser. Enfin, la complexité de fonctionnement des nappes souterraines complique la détermination à la fois de l'institution (à l'échelle d'une nappe, d'un ou plusieurs villages?) et des règles de fonctionnement de cette institution. Certains auteurs, tels Narendranath et al. [14] proposent de se dégager des limites géographiques, naturelles ou administratives, en créant des associations autour de l'utilisation d'un générateur électrique. La réduction de la consommation d'énergie aurait alors un effet indirect sur l'utilisation des pompes.

\section{Gestion concertée entre différentes parties prenantes}

Le troisième type de solutions intègre davantage la notion de gestion concertée entre groupes d'usagers, autorités gouvernementales, ONG pour trouver de nouvelles solutions: une combinaison entre l'approche «top-down » où les règles sont imposées par les instances dirigeantes et l'approche "bottom-up » où des organisations locales sont impulsées par des ONG, doit être trouvée $[14,32]$. Pour cela, il faut dépasser deux visions économiques antagonistes, l'une reposant sur l'intervention publique (réglementation, taxe) et l'autre sur la marchandisation de l'eau souterraine, comme le montre le cadre d'analyse présenté par Petit [33] qui suggère une approche fondée sur la coordination des actions individuelles, des actions collectives d'intérêt public impulsées par l'État et des actions collectives communautaires servant les intérêts de la communauté d'usagers. Le changement d'échelle est alors indispensable: il faut pouvoir intégrer les contraintes locales dans un ensemble environnemental plus vaste, comprendre les interactions hydrauliques entre eaux de surface et eaux souterraines, entre amont et aval au niveau d'un bassin-versant, entre zones urbaine et périurbaine ou industrielle et rurale, approche que les travaux sur la gestion intégrée de l'eau ont développée [34].
Plusieurs éléments font défaut aujourd'hui pour la mise en œuvre d'une gestion concertée de l'eau souterraine en Inde : gestion séparée des eaux de surface et des eaux souterraines empêchant de considérer l'eau comme une ressource unifiée, fragmentation de la gestion de l'eau entre différents ministères et organisations, confusion entourant les droits de propriété, absence de dispositif performant du suivi des niveaux d'eau [4]. Or, l'information à donner aux usagers sur le cycle de l'eau, les quantités d'eau pompées, celles rechargées dans les nappes ${ }^{10}$, etc. semble une étape indispensable pour que les utilisateurs prennent conscience de la nécessité de modifier les pratiques d'irrigation et $s^{\prime}$ impliquent dans un nouveau mode de régulation de l'exploitation de l'eau souterraine.

\section{Conclusion}

Une course effrénée à l'eau souterraine s'observe : les agriculteurs pompent tant et plus, et investissent dans des forages toujours plus profonds au risque de mettre les nappes en péril. Les écarts socioéconomiques sont accentués car les premières victimes sont les petits paysans. L'absence de régulation de l'utilisation de cette eau est frappante et montre, dans les conditions actuelles d'exploitation et de demande en eau, les limites du libre accès. La nécessité de changer les habitudes prises depuis 40 ans est indispensable. La manière de réguler cet accès reste à trouver: "L'heure est à la recherche de solutions innovantes qui doivent être spécifiques à l'environnement d'une région intégrant les conditions sociopolitiques et économiques des agriculteurs» [14]. Certains voient la solution dans une définition plus précise des droits de propriété, tandis que d'autres préconisent une nouvelle gestion, soit communautaire - qui a le défaut de ne pas considérer l'espace hydraulique dans son ensemble - soit concertée, caractéristique des nouvelles formes de gouvernance. Tous les éléments nécessaires à la bonne réalisation de cette dernière ne sont toutefois pas encore réunis, notamment ceux qui doivent être mis en place par l'État. Gestions communautaire ou concertée devraient avoir pour principal impact la prise de conscience collective de la nécessité d'extraire moins d'eau des aquifères et ainsi de faciliter l'application des lois de régulation.

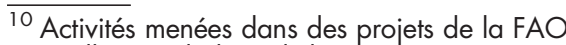
en Andhra Pradesh, et de l'ONG Water Harvest d'Auroville au Tamil Nadu. Ces projets sont toutefois trop récents pour avoir un retour sur leur impact.
} 


\section{Références}

1. Shah T. Community management of groundwater resources: an appropriate response to groundwater overdraft in India? IWMI-Tata Water Policy 2005 : (4) : 11 p.

2. IWMI-Tata Water Policy Program. The Socio-Ecology of Groundwater in India. Water Policy Briefing 2002: (4): 8 p. >http:// www.iwmi.cgiar.org/publications/Water_Policy_ Briefs/PDF/wpb04.pdf

3. Government of India. Mid-term appraisal of 10th Five year Plan (2002-2007). 2005. http://planningcommission.nic.in/midterm/midtermapp.html.

4. Petit $O$. La gouvernance des eaux souterraines et de l'irrigation en Inde. Entre régulation publique et participation locale des usagers. In : Froger G, Mainguy C, Brot J, Gérardin H, eds. Quels acteurs pour quel développement? Paris : GEMDEV-Karthala, 2005.

5. Calvo-Mendieta I. Conflits d'usage dans la gestion des ressources en eau: analyse territoriale des modes de régulation. Actes de la journée d'étude "les territoires de l'eau», Université d'Artois, Arras, 26 mars 2004.

6. Unesco. Une pénurie croissante. Le courrier de l'Unesco. 2001. www.unesco.org/courier/ 2001_10/fr/doss02.htm

7. Bon E. Le drame des communaux. Appropriation publique, privée, commune ou accès libre aux espaces-ressources naturels indiens? Thèse de doctorat en sciences économiques, université de Paris-X Nanterre, 2005.

8. Government of India. Ground water management and ownership (report of the experts). New Delhi : Planning Commission, 2007.

9. Bromley DW. Environment and Economy. Property Rights and Public Policy. Cambridge (Massachusetts) : Basil Blackwell, 1991.

10. Prakash A. The Dark zone. Groundwater irrigation, politics and social power in North Gujarat. New Delhi : Orient Longman, 2005.

11. Janakarajan S. In search of tanks : some hidden facts. Economic and Political Weekly 1993 ; June 26 : A53-60.
12. Mosse D. The rule of water. Statecraft, Ecology and Collective Action in South India. New Delhi : Oxford University Press, 2003.

13. Janakarajan S, Moench M. Are wells a potential threat to farmers' wellbeing? The case of deteriorating groundwater irrigation in Tamil Nadu. Madras Institute of Development Studies (MIDS) working paper, $n^{\circ} 174$. Chennai : MIDS, 2002.

14. Narendranath G, UmaS, Reddy KR. To free or not to free power. Understanding the context of free power to agriculture. Economic and Political Weekly $2005 ; 40$ : 5561-70.

15. Shah T. Groundwater markets and irrigation development. Political economy and practical policy. Bombay : Oxford University Press, 1993.

16. Palanisami K. Techno-economic feasibility of groundwater over-exploitation in Tamil Nadu. Annual Partners' meet 2002 of IWMI-Tata water policy research program, Colombo, 2002. Colombo : IWMI, 2002.

17. Kumar MD. Groundwater management in India. Physical, institutional and policy alternatives. New Delhi : Sage publications, 2007.

18. Narayanamoorthy A, Deshpande RS. Where water seeps! Towards a new phase in India's Irrigation Reforms. New Delhi : Academic Foundation, 2005.

19. Sinha SK, Talati J. Impact of system of rice intensification (SRI) on rice yields:results of a new sample. study in Purulia district, India. Water Policy Research 2005; 20 : 11 p.

20. Trébuil G, Hossain M. Le riz. Enjeux écologiques et économiques. Collection Mappemonde. Paris : Belin, 2004.

21. Tamil Nadu Water Supply and Drainage (TWAD). Democratisation of water management. 2005. www.twadboard.com/water_democ.pdf

22. Rajagopal A, Janakarajan S. Water-rights and participatory irrigation management in India: the case of surface water sector in Tamil Nadu state. www.water-2001.de/datenbank/ 46076235.41368.14/WATER \%20RIGHT \%20PAP.doc.
23. Aubriot $O$. Baisse des nappes phréatiques en Inde du Sud : forte demande sociale et absence de gestion de la ressource. Géocarrefour 2006 ; 81 : 83-96.

24. Dubash NK. Tubewell capitalism: groundwater development and agrarian change in Gujarat. Delhi : Oxford University Press, 2002.

25. Viswanathan S. Battling coke in Sivaganga. Frontline 2003; 07 June.

26. Honneger A, Bravard JP. La pénurie de l'eau : donnée naturelle ou question sociale? Géocarrefour $2005 ; 80$ : 257-60.

27. Honneger A, Bravard JP. La pénurie de l'eau : donnée naturelle ou question sociale? Géocarrefour 2006 ; 81 : 3-4.

28. Coste N, Ploumpidis N, Anupama K, Aubriot $O$, Gunnell Y. Bittersweet water. Irrigation practices and modern challenges in South India. Trilingual DVD (Eaux douces, Eaux amères- Thaneer: inippum kacappum). (Publications Hors Série 5). Pondicherry: French Institute of Pondicherry, 2007.

29. Shah T. Groundwater management in India. In : Anil A, ed. The challenge of the balance: environmental economics in India. Proceedings of the National Environment and Economics Meeting, January 1994). Delhi: Centre for Science and Environment, 1997.

30. Shiva V. Turning scarcity into abundance. Yesmagazine 2004 ; winter issue.

31. CulletP. Water law reforms. Analysis of recent developments. Journal of the Indian Law Institute 2006 ; 48 : 206-31.

32. Kemper KE. Rethinking groundwater management. In: Figuères CM, Tortajada $C$, Rockström J, eds. Rethinking water management. Innovative approaches to contemporary issues. London : Earthscan Publications, 2003.

33. Petit $O$. La surexploitation des eaux souterraines: enjeux et gouvernance. Natures Sciences Sociétés $2004 ; 12$ : 146-56.

34. Molden D. Water for food, water for life: A comprehensive assessment of water management. London; Colombo: Earthscan; IWMI, 2007. 